\title{
Revealing acquired resistance mechanisms of kinase- targeted drugs using an on-the-fly, function-site interaction fingerprint approach
}

Zheng Zhao ${ }^{1}$ and Philip E. Bourne*,1,2

1. Department of Biomedical Engineering, University of Virginia, Charlottesville, Virginia 22904, United States of America

2. School of Data Science, University of Virginia, Charlottesville, Virginia 22904, United States of America

*Corresponding author

Philip E. Bourne: peb6a@virginia.edu 


\section{Table of Contents}

1. Aligning the Crizotinib-binding wild/mutated X-ray structures.............................................................

2. Equilibrating the wildtype/mutated drug-bound ALK systems and determining the reaction coordinates ...4

3. Sampling the drug binding process of each system. The dynamic change of ALK kinase in every system was shown.

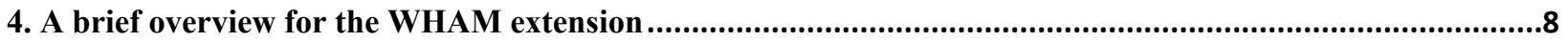

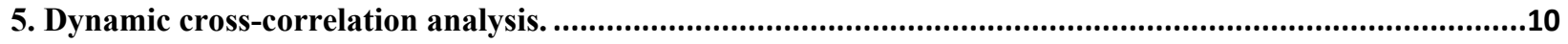

6. Equilibrating the wildtype/mutated Alectinib-bound ALK systems and determining the reaction

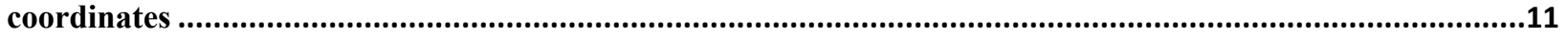

7. Table S1. Interaction fingerprints of initial ALK-drug wildtype(wt)/mutated(mt) complex structures.......13

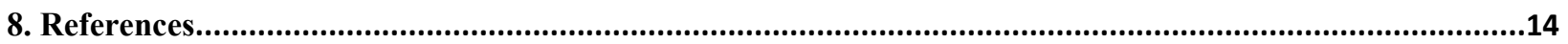




\section{Aligning the Crizotinib-binding wild/mutated X-ray structures}

The alignment was carried out using the PDB online database ${ }^{1}$. We took two complex structures, 2xp2 for the wild-type and 2yfx for the L1196M mutated ALK-Crizotinib complex. Then, using the application of Sequence \& Structure Alignment

(https://www.rcsb.org/pages/analyze features\#Sequence ), we input the A chain of the two structures and applied the jFATCAT structure alignment method ${ }^{2}$.

The ligands overlapped completely and the RMSD for the two protein structures is $0.39 \mathrm{~A}^{2}$, implying highly similar structures (Figure S1).

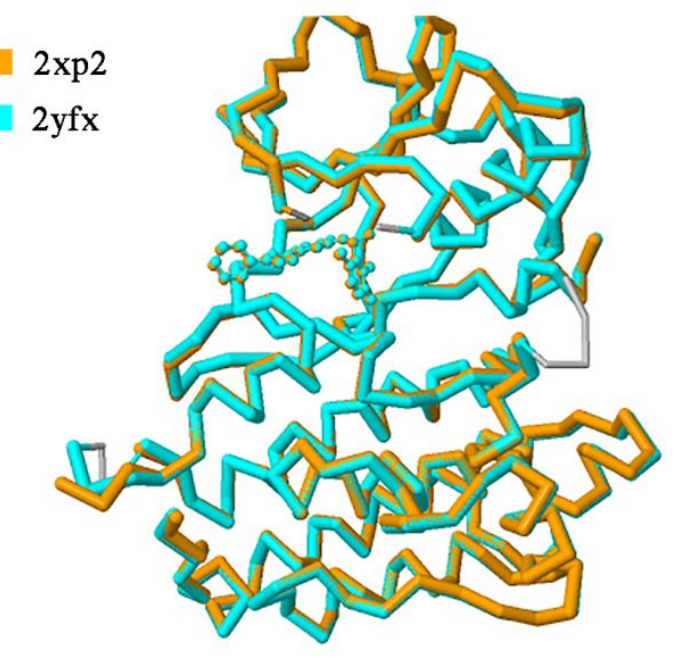

Figure S1. The alignment of the wildtype and mutated ALK-Crizotinib complex structures (PDB ids: $2 x p 2$ and $2 y f x)$. 


\section{Equilibrating the wildtype/mutated drug-bound ALK systems and determining the}

reaction coordinates

Before carrying out umbrella sampling free energy calculations all ALK systems were equilibrated by running 150ns MD simulations and the corresponding $\mathrm{C} a$-RMSDs are shown in Figure S2. To determine the common reaction coordinates, we analyzed the respective equilibrated system by calculating the Ca-RMSF of every system (Figure S3). Based on the inflexibility of every residue at the binding site, we chose the rigid residues to build the reaction coordinates (Figure S4). Then we chose the distance between the center of mass of the Ca atom of these residues (including residue 1169-1172, 1196-1199, and 1267-1268; green) and the center of mass of ligand core structure (red) as the reaction coordinates to represent the process of drug binding.

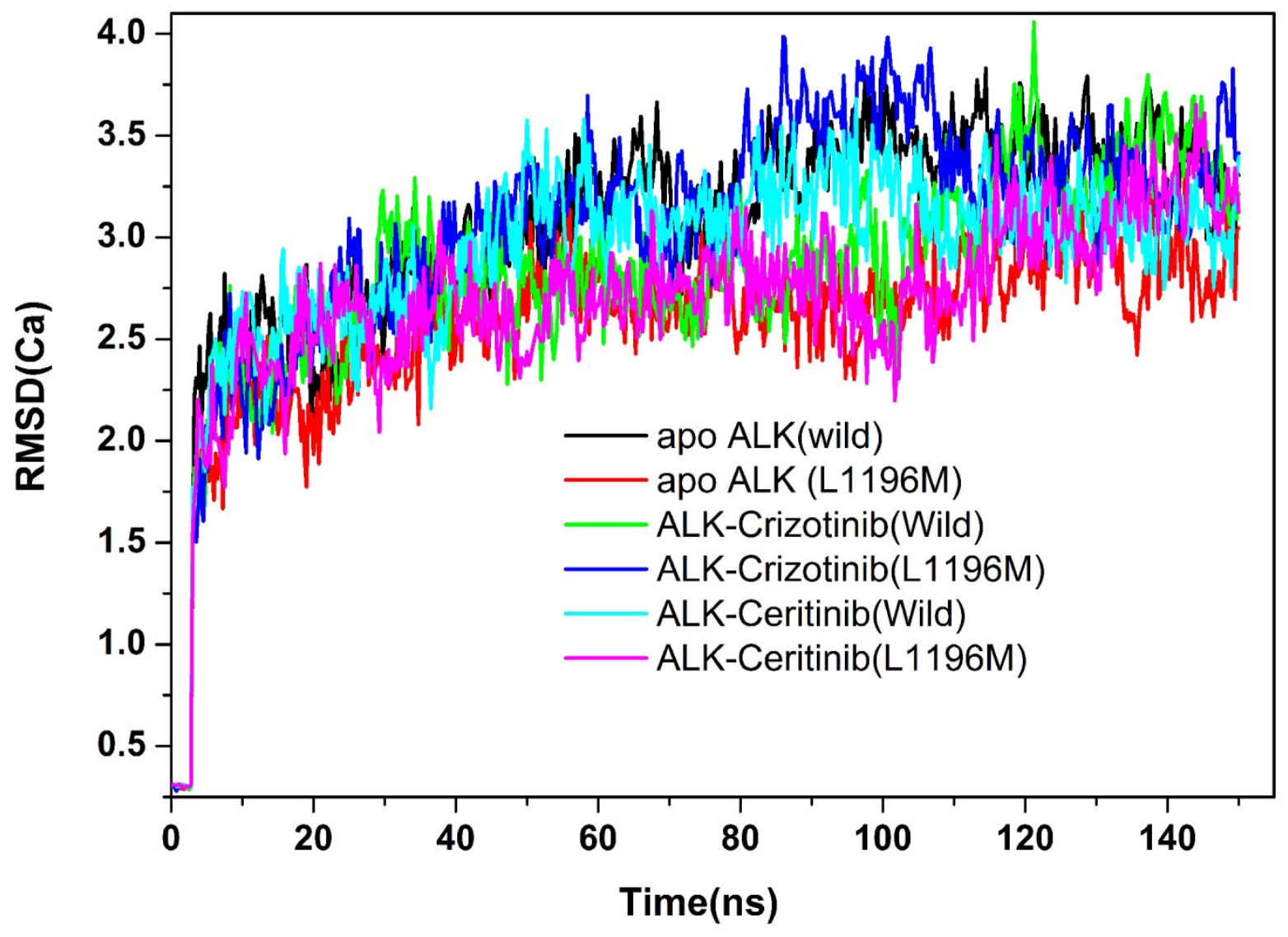


Figure S2. Ca-RMSDs of all ALK systems.

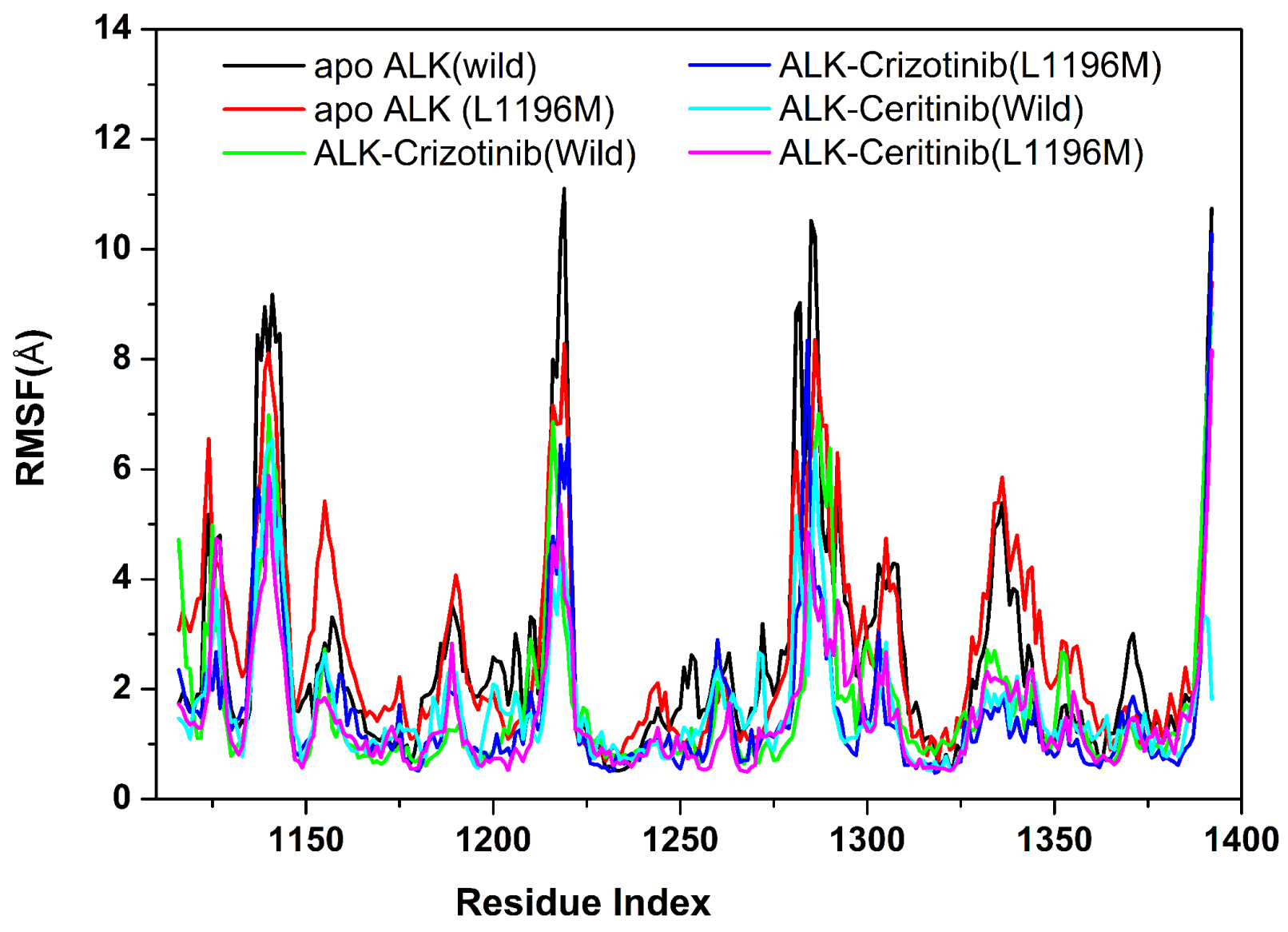

Figure S3. Ca-RMSFs of all ALK systems. 

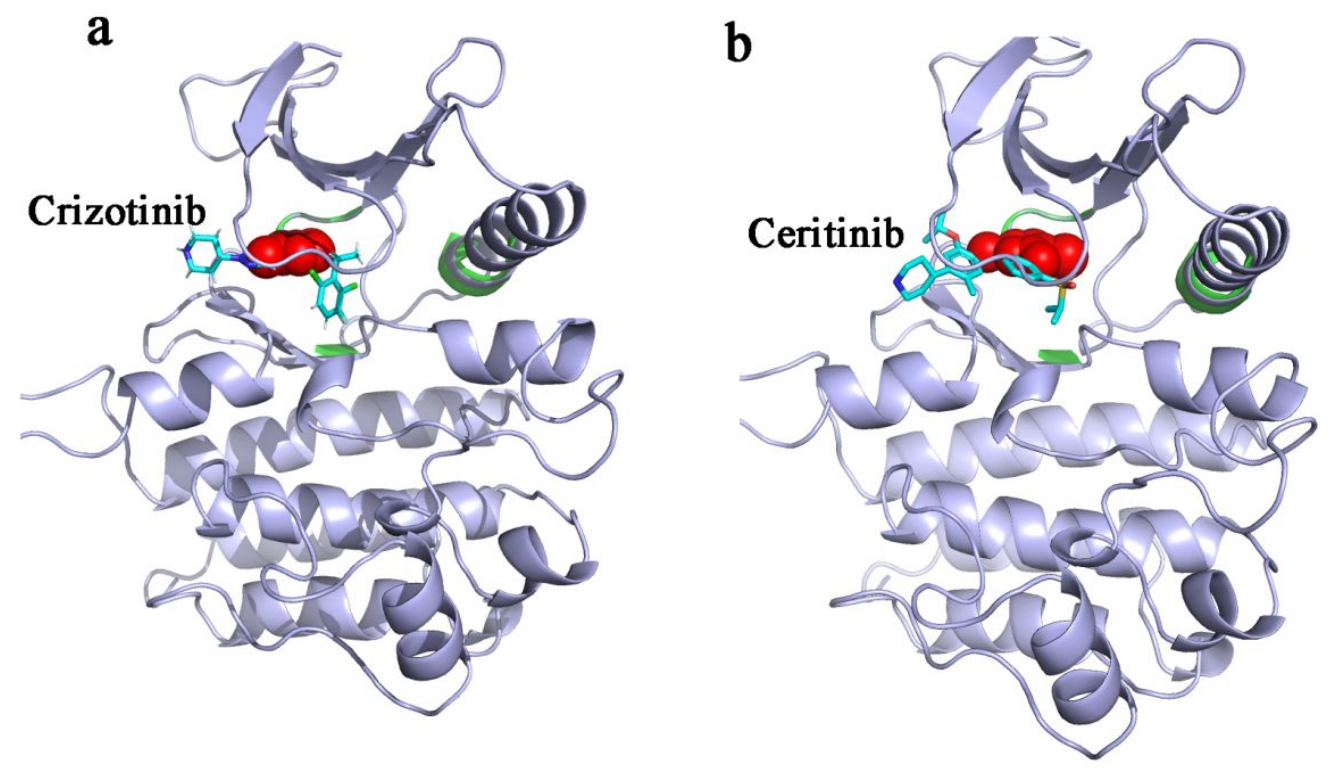

Figure S4. Definition of the reaction coordinates for different drug-ALK complexes. The distances between the center of mass of the $\mathrm{Ca}$ atom of these residues, including residue 11691172, 1196-1199, and 1267-1268 (green color) and the center of mass of ligand core structure (red), are used as the reaction coordinates. 
3. Sampling the drug binding process of each system. The dynamic change in the ALK kinase for every system is shown.

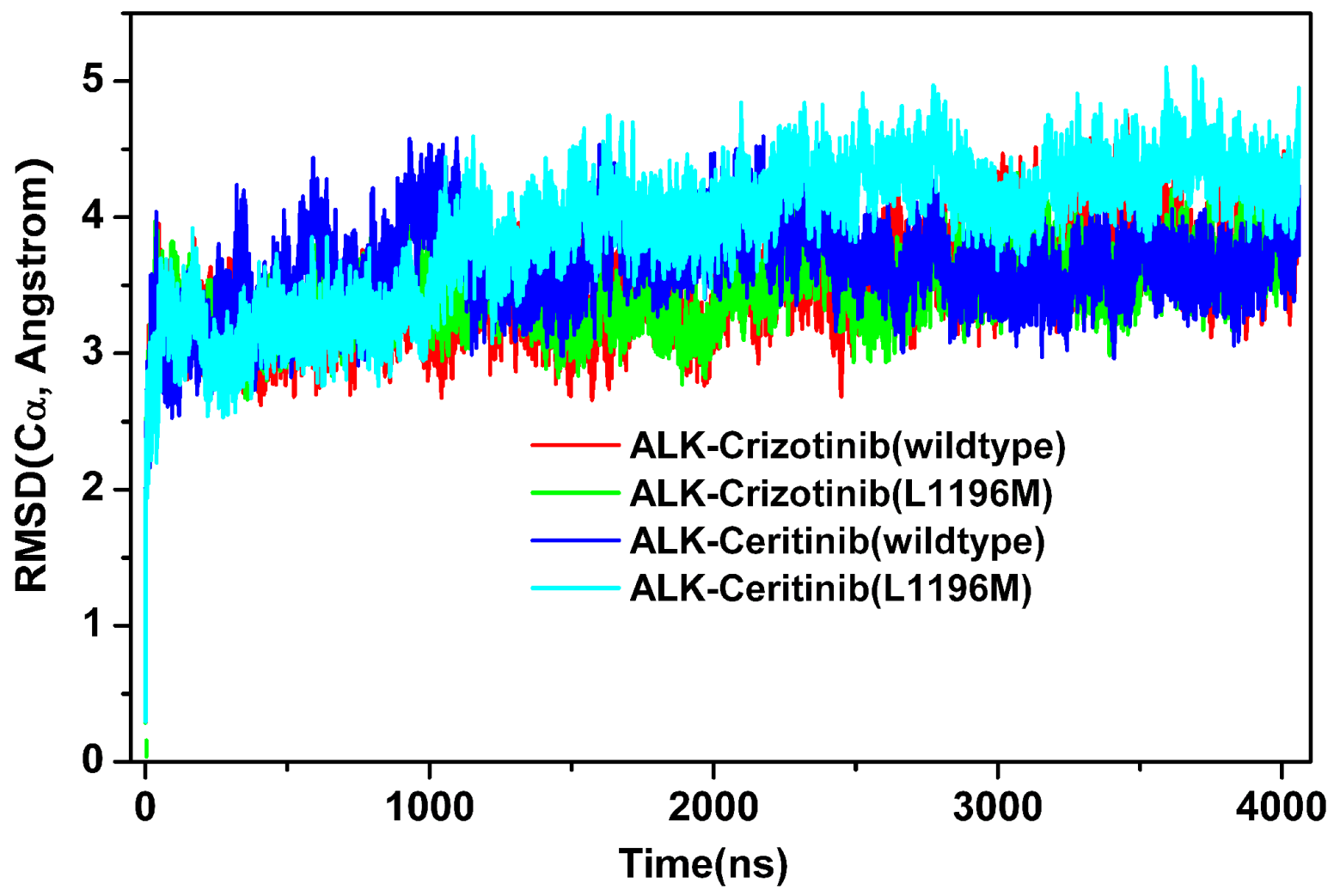

Figure S5. RMSDs of each drug-binding ALK system using Ca atoms with 4000ns sampling simulations (160ns (each window) $\times 25$ windows).

\section{A brief overview for the WHAM extension}

In umbrella sampling (US) with a biased system Hamiltonian $H i$,

$$
H i=H O+V i
$$

$H O$ is the system Hamiltonian and $V i$ is the bias potential added. The added bias potential will improve the sampling frequency for fields of high energy. Ideally $V i$ should be the negative of the potential of mean force $F i$. Fi is related to Boltzmann distribution, i.e.,

$$
F i=-k T \ln \rho 0(\lambda)
$$


where $\lambda$ is the reaction coordinate, and $\rho 0$ is the probability distribution. At the beginning the $\rho 0$ is not known, however $\rho 0$ can be written in terms of non-Boltzmann probabilities $\rho b i a s$ after a set of biased sampling with bias potential $\mathrm{V}_{\mathrm{i}}$, i.e.,

$$
\rho 0=e^{(V i(\lambda)-F i) / k B T} * \rho \operatorname{bias}(\lambda)
$$

which can be rewritten as:

$$
e^{-F i / k B T}=\int e^{-V i(\lambda) / k B T} \cdot \rho 0(\lambda) d \lambda=<e^{-V i(\lambda) / k B T}>
$$

Where $\rho 0$ cannot be calculated directly. WHAM is an alternative approach designed to determine the total probability distribution $\rho 0$. WHAM is defined as follows ${ }^{3-4}$.

$$
\rho 0(\lambda)=\sum_{i=1}^{N} \frac{n i}{\sum_{j=1}^{N} n j e^{-(V i(\lambda)-F i) / k B T}} \rho_{i}^{\text {bias }}(\lambda)
$$

Where, $n i$ is the number of samples in the $i t h$ window. There are non-linear interdependent variables in equations (4) and (5), which can be solved self-consistently. $V i$ can be arbitrary function, such as the harmonic restraint formation as we used in the work,

$$
V i=k i \cdot\left(\lambda^{\prime} i-\lambda i\right) 2
$$

Where $k i=100 \mathrm{kcal}^{\mathrm{m}} \mathrm{mol}^{-1} .^{-1}$ 
5. Dynamic cross-correlation analysis.

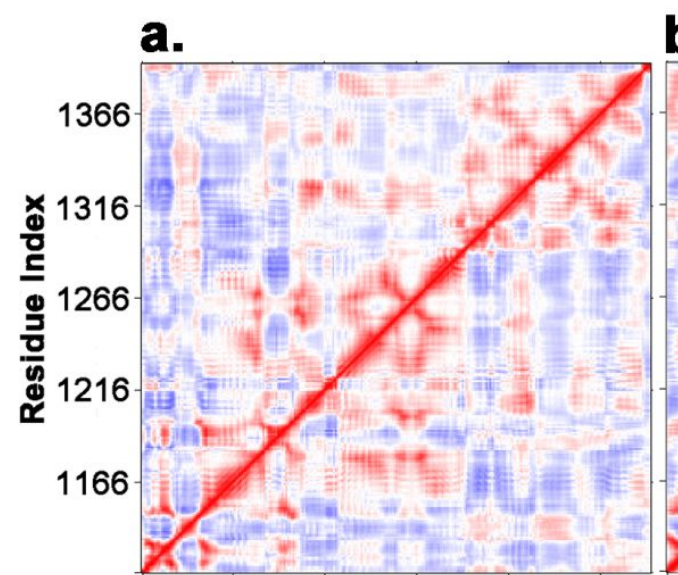

b.

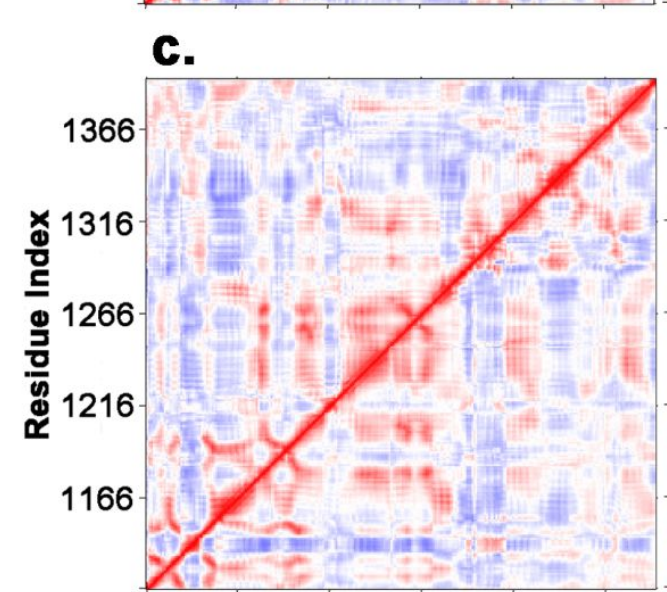

d.

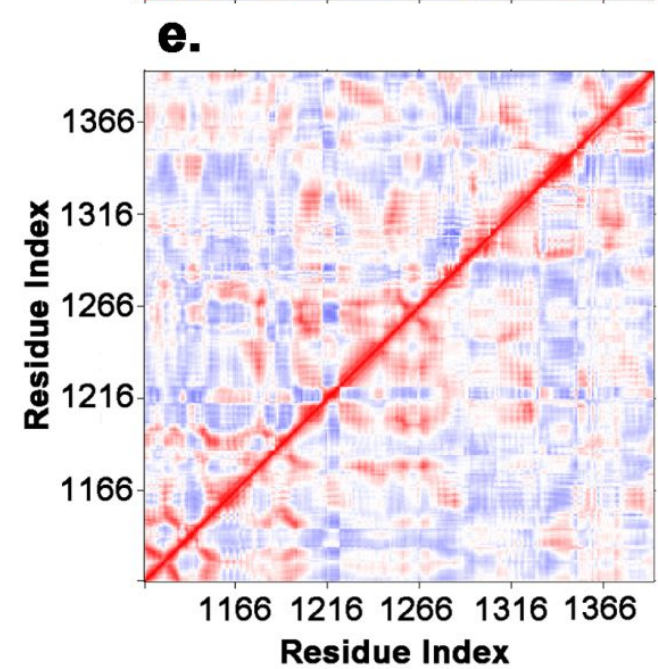

f.
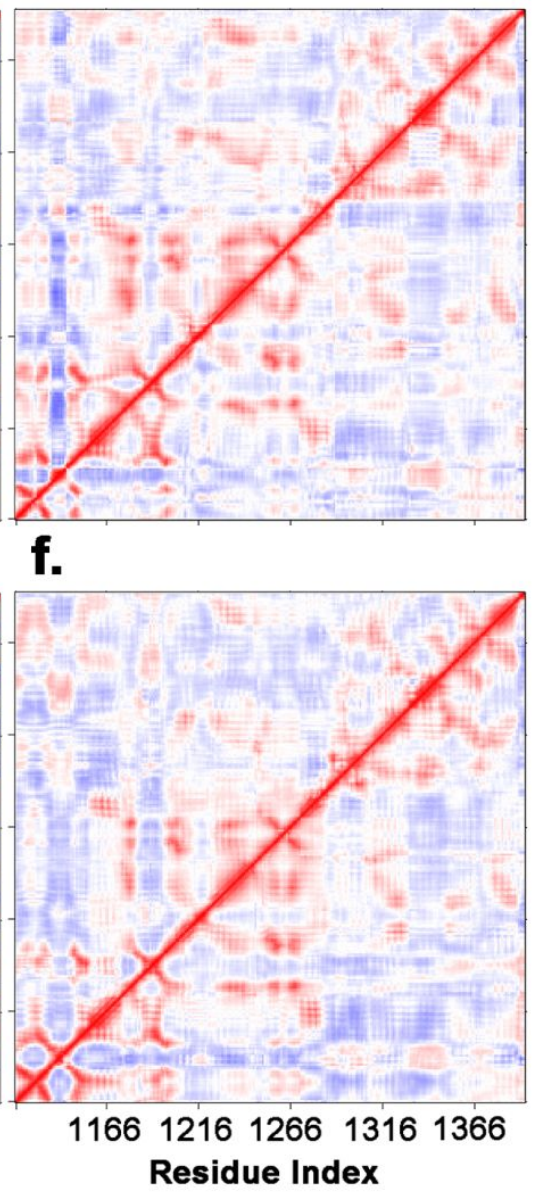
Figure S6. Comparisons (upper triangle vs lower triangle) of the dynamic cross-correlation maps from two different 100ns MD simulations on the same system: (a) wild-type ALK system; (b) L1196M mutated ALK system; (c) Crizotinib-bound wild-type ALK system; (d) Crizotinibbound L1196M mutated ALK system; (e) Ceritinib-bound wild-type ALK systems; and (f) Ceritinib-bound L1196M mutated ALK system.

\section{Equilibrating the wildtype/mutated Alectinib-bound ALK systems and determining the reaction coordinates}

Before carrying out umbrella sampling for the free energy calculations, the Alectinib-bound wildtype/mutated ALK systems were equilibrated by running 150ns MD simulations with the corresponding Ca-RMSDs shown in Figure S7. To determine the common reaction coordinates, we analyzed the respective equilibrated system by calculating the Ca-RMSF of every system (Figure S8). Based on the inflexibility of every residue at the binding site, we choose the rigid residues to build the reaction coordinates (Figure S9). Thus, we chose the distance between the center of mass of the $\mathrm{Ca}$ atom of these residues (including residue 1169-1172, 1196-1199, and 1267-1268; green) and the center of mass of the ligand core structure (red) as the reaction coordinates to indicate the process of drug binding. 


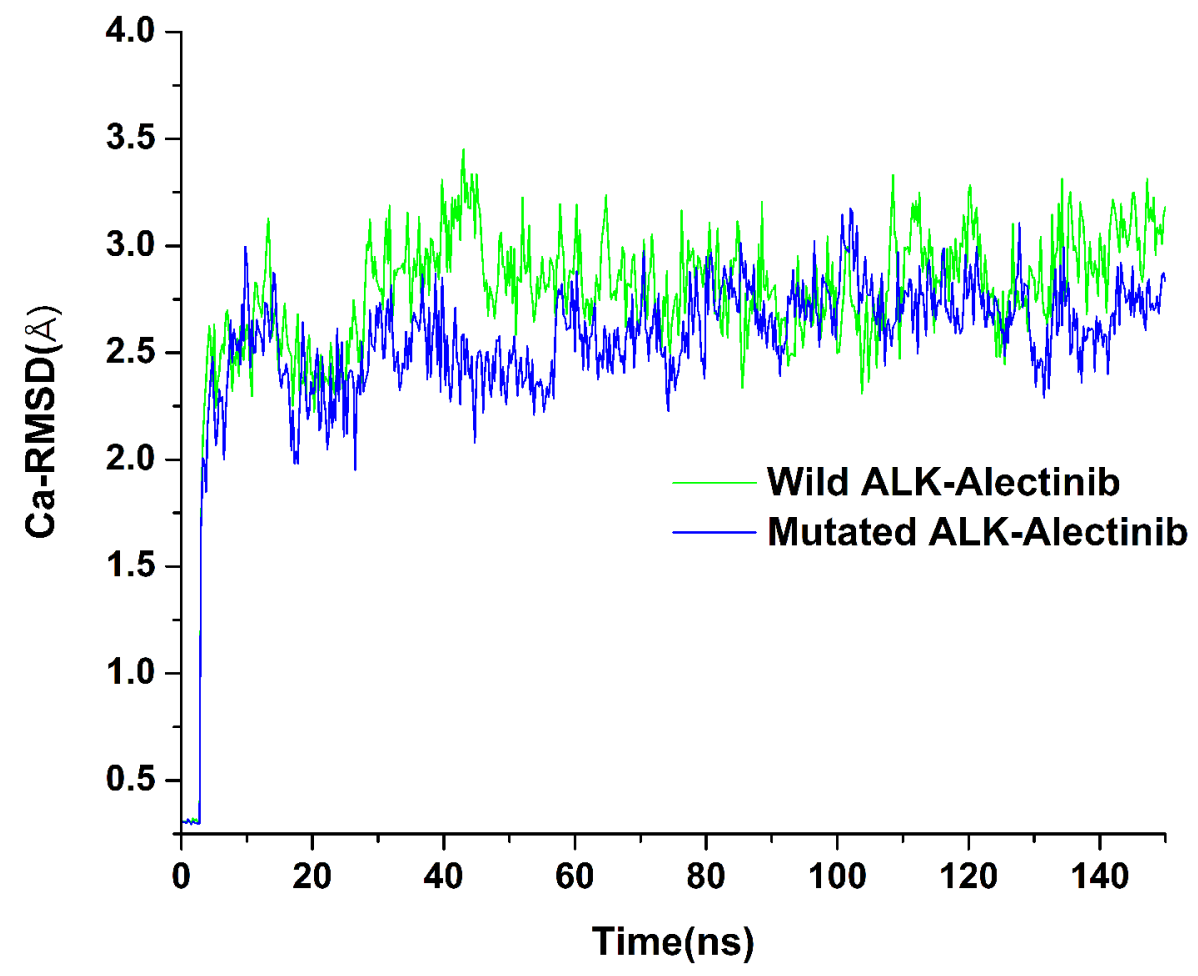

Figure S7. RMSDs of Alectinib-bound ALK systems using Ca atoms.

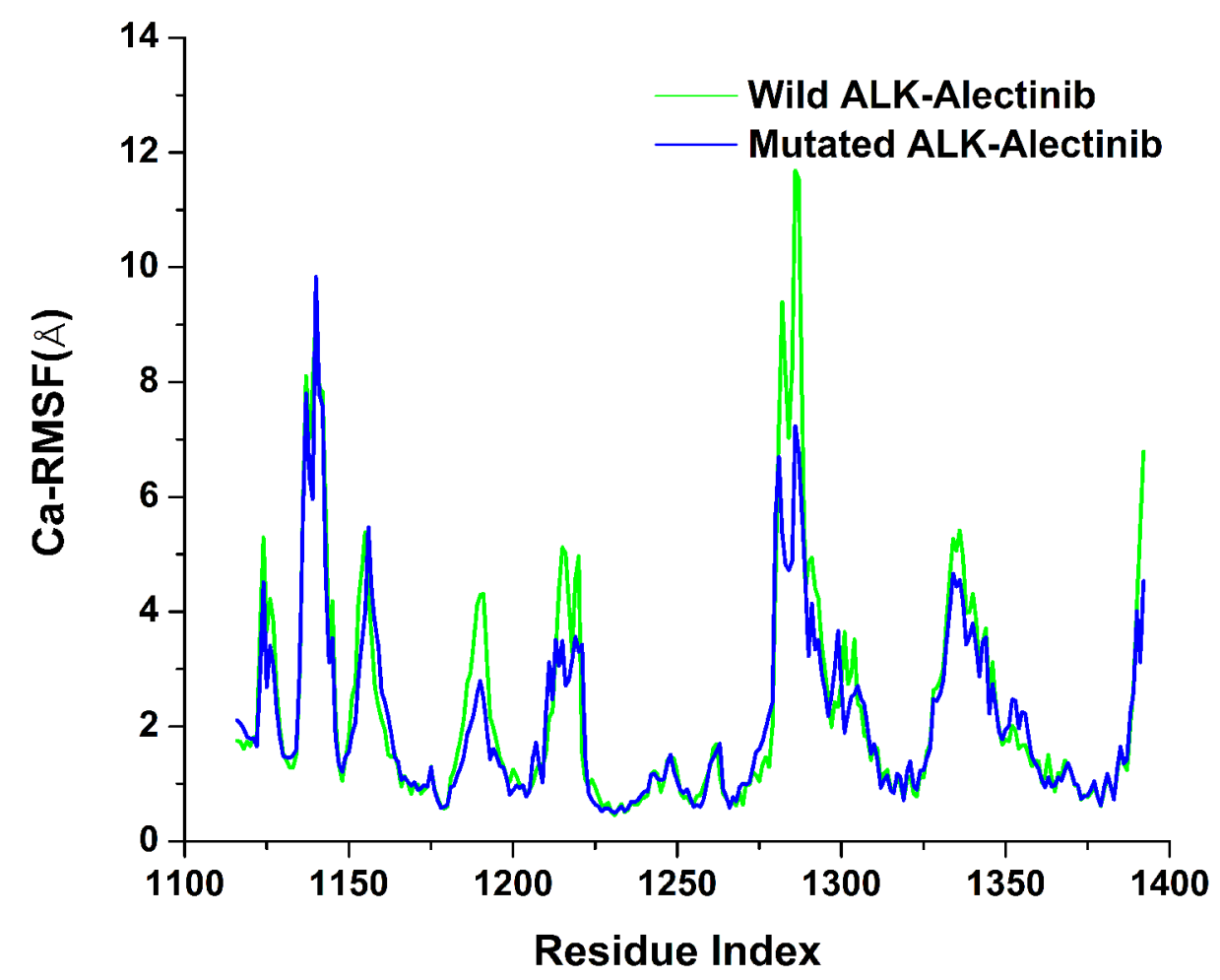

Figure S8. RMSFs of Alectinib-bound ALK systems using Ca atoms. 


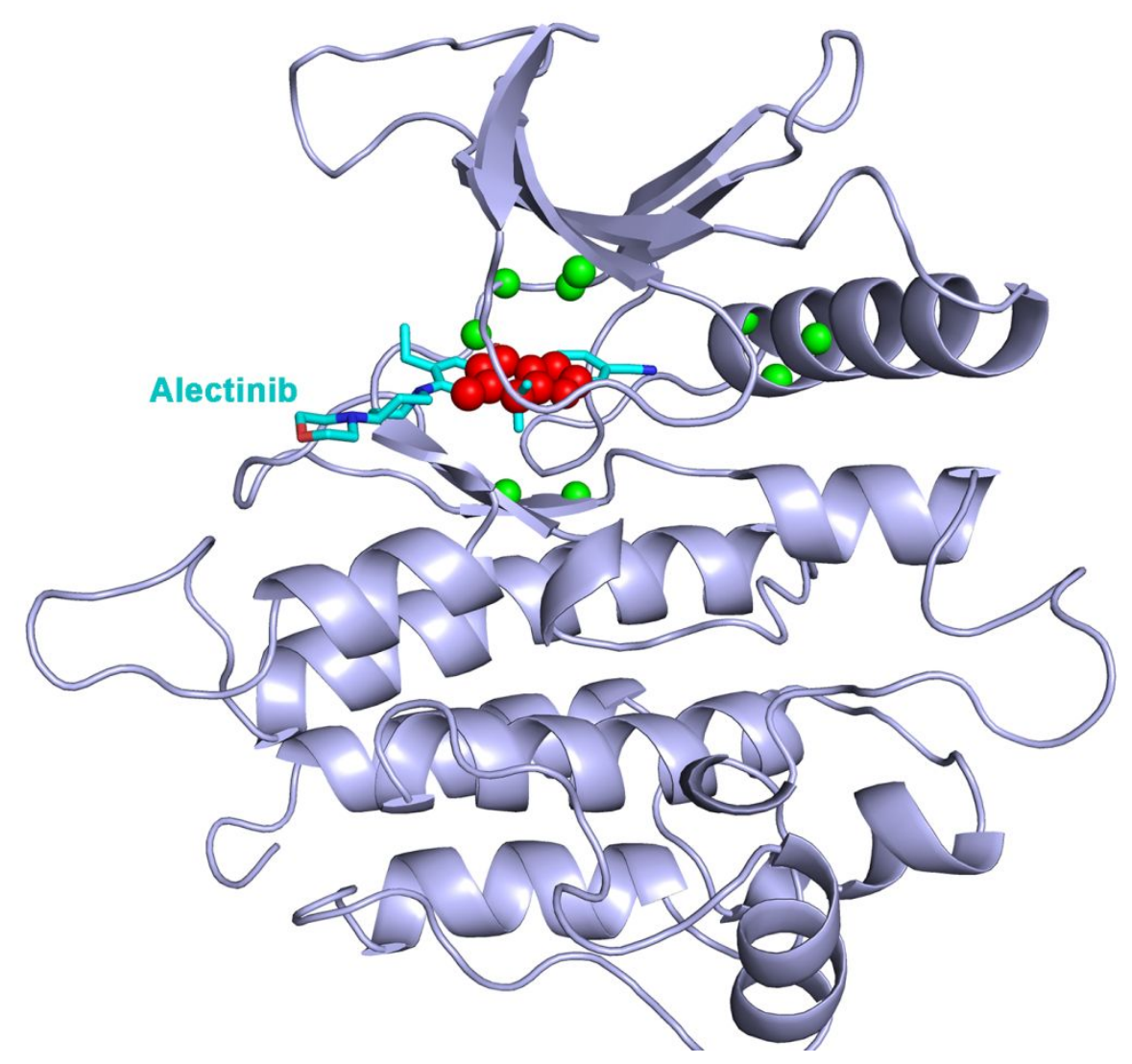

Figure S9. Definition of the reaction coordinates for the Alectinib-bound ALK complex. The distance between the center of mass of the Ca atom of these residues including residue 1169-1172, 1196-1199, and 1267-1268( in green color) and the center of mass of the ligand core structure (in red color) is defined as the reaction coordinates.

\section{Table S1. Interaction fingerprints of initial ALK-drug wildtype(wt)/mutated(mt)}

\section{complex structures.}

\begin{tabular}{|l|l|l|l|l|l|l|l|}
\hline \multicolumn{2}{|l|}{ ALK(wt)-Crizotinib } & \multicolumn{2}{l|}{ ALK(mt)-Crizotinib } & \multicolumn{2}{l|}{ ALK(wt)-Ceritinib } & \multicolumn{2}{l|}{ ALK(mt)-Ceritinib } \\
\hline Residues & IFP & Residues & IFP & Residues & IFP & Residues & IFP \\
\hline LEU1122 & 1000000 & LEU1122 & 1000000 & LEU1122 & 1000000 & LEU1122 & 1000000 \\
\hline VAL1130 & 1000000 & VAL1130 & 1000000 & GLY1123 & 1000000 & GLY1123 & 1000000 \\
\hline ALA1148 & 1000000 & ALA1148 & 1000000 & VAL1130 & 1000000 & VAL1130 & 1000000 \\
\hline LYS1150 & 1000000 & LYS1150 & 1000000 & GLU1132 & ------ & GLU1132 & 1000000 \\
\hline LEU1196 & 1000000 & MET1196 & 1000000 & ALA1148 & 1000000 & ALA1148 & 1000000 \\
\hline GLU1197 & 0000100 & GLU1197 & 0000100 & LYS1150 & ------- & LYS1150 & 0001000 \\
\hline
\end{tabular}




\begin{tabular}{|l|l|l|l|l|l|l|l|}
\hline LEU1198 & 1000000 & LEU1198 & 1000000 & LEU1196 & 1000000 & MET1196 & 1000000 \\
\hline MET1199 & 1001000 & MET1199 & 1001000 & LEU1198 & 1000000 & LEU1198 & 1000000 \\
\hline GLY1202 & 1000000 & GLY1202 & 1000000 & MET1199 & 1001100 & MET1199 & 1001100 \\
\hline ASP1203 & 1000000 & ASP1203 & 1000000 & ALA1200 & 1000000 & ALA1200 & 1000000 \\
\hline ASN1254 & 1000000 & ASN1254 & 1000000 & GLY1202 & 1000000 & GLY1202 & 1000000 \\
\hline LEU1256 & 1000000 & LEU1256 & 1000000 & ASP1203 & 100000 & ASP1203 & 1000000 \\
\hline GLY1269 & 1000000 & GLY1269 & 1000000 & SER1206 & 1000000 & SER1206 & 1000000 \\
\hline ASP1270 & 1000000 & ASP1270 & 1000000 & LEU1256 & 1000000 & LEU1256 & 1000000 \\
\hline & & & & ASP1270 & 1000000 & ASP1270 & 1000000 \\
\hline
\end{tabular}

The initial crystal structures with PDB ids: 2xp2, 2yfx and 4mkc are used to encode ALK(wt)Crizotinib, ALK(mt)-Crizotinib, and ALK(wt)-Ceritinib systems, respectively. The Ceritinib$\operatorname{ALK}(\mathrm{mt})$ complex is from the docked model as described in the Method section.

\section{References}

1. Prlic, A.; Bliven, S.; Rose, P. W.; Bluhm, W. F.; Bizon, C.; Godzik, A.; Bourne, P. E., Precalculated protein structure alignments at the RCSB PDB website. Bioinformatics 2010, 26 (23), 2983-2985.

2. Ye, Y.; Godzik, A., Flexible structure alignment by chaining aligned fragment pairs allowing twists. Bioinformatics 2003, 19 Suppl 2, ii246-55.

3. Kumar, S.; Bouzida, D.; Swendsen, R. H.; Kollman, P. A.; J.M.., R., The Weighted Histogram Analysis Method for Free Energy Calculations on Biomolecules .I. The Method. $J$. Comput. Chem. 1992, 13 (8), 1011-1021.

4. Dimelow, R. J.; Bryce, R. A.; Masters, A. J.; Hillier, I. H.; Burton, N. A., Exploring reaction pathways with transition path and umbrella sampling: application to methyl maltoside. $J$. Chem. Phys. 2006, 124 (11), 114113-114128. 